\title{
CCL25 promotes the migration and invasion of non-small cell lung cancer cells by regulating VEGF and MMPs in a CCR9-dependent manner
}

\author{
YUXU NIU ${ }^{1 *}$, DONGFANG TANG ${ }^{1 *}$, LIWEN FAN $^{1}$, WEN GAO $^{1}$ and HUI LIN ${ }^{2}$ \\ ${ }^{1}$ Department of Thoracic Surgery, Shanghai Key Laboratory of Clinical Geriatric Medicine, Huadong Hospital, \\ Fudan University, Shanghai 200040; ${ }^{2}$ Department of Thoracic Surgery, The People's Hospital of \\ Guangxi Zhuang Autonomous Region, Nanning, Guangxi 530021, P.R. China
}

Received August 4, 2019; Accepted March 5, 2020

DOI: $10.3892 /$ etm.2020.8635

\begin{abstract}
The CC chemokine receptor 9 (CCR9) and its natural secreted ligand CC motif chemokine ligand 25 (CCL25) have been implicated in cancer metastasis. However, their metastatic potential in non-small cell lung cancer (NSCLC) remains unclear. In the present study, immunohistochemistry was used to detect the expression and localization of CCR9, vascular endothelial growth factor (VEGF), matrix metalloproteinase (MMP)-1 and MMP-7 in lung cancer tissue and adjacent normal tissue. The association between the expression of CCR9 and clinical variables was also examined. Reverse transcription-quantitative PCR and western blotting were conducted to detect the expression of VEGF-C, VEGF-D, MMP-1 and MMP-7 in lung cancer cell lines (A549 and SK-MES-1). Migration and invasion assays were conducted to examine cell migration and invasion. Survival and mutation analysis were conducted using published datasets. The expressions of CCR9,
\end{abstract}

Correspondence to: Professor Wen Gao, Department of Thoracic Surgery, Shanghai Key Laboratory of Clinical Geriatric Medicine, Huadong Hospital, Fudan University, 221 West Yan'an Road, Jing'an, Shanghai 200040, P.R. China

E-mail: gaowenchest@163.com

Professor Hui Lin, Department of Thoracic Surgery, The People's Hospital of Guangxi Zhuang Autonomous Region, 6 Taoyuan Road, Qingxiu, Nanning, Guangxi 530021, P.R. China

E-mail: linhuichest@163.com

*Contributed equally

Abbreviations: NSCLC, non-small cell lung cancer; CCR9, CC chemokine receptor 9; CCL25, CC motif chemokine ligand 25; VEGF-C, vascular endothelial growth factor-C; VEGF-D, vascular endothelial growth factor-D; MMP-1, matrix metalloproteinase-1; MMP-7, matrix metalloproteinase-7; SCC, squamous cell carcinoma; $\mathrm{AC}$, adenocarcinoma; OS, overall survival

Key words: non-small cell lung cancer, chemokine receptor 9, CC motif chemokine ligand 25 , metastasis
VEGF, MMP-1 and MMP-7 were upregulated in cancer tissue, compared with adjacent normal tissue (all $\mathrm{P}<0.05$ ). Patients with lower expression of CCR9 or CCL25 had better overall survival (OS) compared with those with higher CCR9 or CCL25 expression ( $\mathrm{P}<0.05$ and $\mathrm{P}=0.05$, respectively). Furthermore, the expressions of VEGF-C, VEGF-D, MMP-1 and MMP-7 were higher in the CCL25-treated cell lines (all $\mathrm{P}<0.05)$, but MMP-7 protein expression was not affected by CCL25 treatment in SK-MES-1 cells ( $\mathrm{P}>0.05)$. Following treatment with CCL25, lung cancer cells demonstrated higher migratory and invasive potential, which could be blocked by the CCR9 antibody $(\mathrm{P}<0.05)$. Survival analysis demonstrated that low expression levels of both CCR9 and CCL25 mRNA indicated favorable OS in patients with NSCLC. Altogether, these results suggested that CCL25 enhanced the phenotype associated with migration and invasion in NSCLC by regulating the expression of VEGF-C, VEGF-D, MMP-1 and MMP-7.

\section{Introduction}

Lung cancer is the leading cause of cancer-related death worldwide, $85 \%$ of which is non-small cell lung cancer (NSCLC) (1), which usually invades the regional or distant lymph nodes, brain and liver $(2,3)$. Metastasis is typically the most common risk of death in NSCLC, which suggests that blocking the migration and invasion of tumor cells might be a possible therapeutic strategy.

A previous study identified that migration and invasion of tumor cells could be regulated by chemokines and their receptors (4). Chemokines, which are a family of small, structurally related heparin-binding proteins classified as $\mathrm{C}, \mathrm{CXC}, \mathrm{CC}$ and $\mathrm{CX} 3 \mathrm{C}$ sub-families $(5,6)$, are essential to the normal and pathologic trafficking of leukocytes. The biological functions of chemokines are mediated via G-protein-coupled receptors, which play an important role in inflammation and leukocyte differentiation. It has been demonstrated that various cell types may express different chemokine receptors, such as $\mathrm{C}-\mathrm{X}-\mathrm{C}$ chemokine receptor type 2 (CXCR2) (7), C-X-C chemokine receptor type 4 (CXCR4) (8), C-C chemokine receptor type 6 (CCR6) (9) and C-C chemokine receptor type 9 (CCR9) (10). 
High expression of CXCR2, CXCR4 and CCR9 with their respective ligands $\mathrm{C}-\mathrm{X}-\mathrm{C}$ motif chemokine $8, \mathrm{C}-\mathrm{X}-\mathrm{C}$ motif chemokine 12 and $\mathrm{CC}$ motif chemokine ligand 25 (CCL25) was associated with tumor metastasis and poor survival in NSCLC (11-13). We previously demonstrated that CCR9 and CCL25 interaction could suppress apoptosis in a phosphoinositide 3-kinase/Akt-dependent model in the NSCLC cell lines A549 and SK-MES-1 (14). However, the exact molecular mechanism of migration and invasion in NSCLC remains elusive.

The present study aimed to examine the potential association between the CCL25/CCR9 axis and key molecules involved in lung cancer cell migration, and to verify further the association between the CCL25/CCR9 axis and key molecules examined (VEGF-C, VEGF-D, MMP-1 and MMP-7) by CCR9 drug treatment experiments. A Kaplan-Meier survival analysis was used to further confirm the association between the CCL25/CCR9 axis and prognosis of lung cancer.

\section{Materials and methods}

Study design. A total of 60 patients with NSCLC who underwent surgery in The Huadong Hospital Affiliated with Fudan University were enrolled in the present study between September 2012 and December 2015. Tumor tissues and adjacent normal tissues were collected and paraffin-embedded. Neither chemotherapy nor immunotherapy was performed prior to surgery. A double pathological diagnosis was performed prior to enrolling the patients in the present study. The median age of these patients was 58 years, with the age ranging from 31 to 76 . Further patient information is presented in Table I. The present study protocol was approved by The Ethics Committee Review Board of The Huadong Hospital Affiliated with Fudan University, and written informed consent was obtained from every participant.

Immunohistochemistry (IHC) assay. Formalin-fixed (10\%, for $8 \mathrm{~h}$ at room temperature), paraffin-embedded tissues were sliced into $4-\mu \mathrm{m}$ sections using a microtome, then successively deparaffinized with xylene, rehydrated in a descending alcohol series and rinsed with PBS followed by high-pressure antigen retrieval in a microwave oven with citrate buffer $\left(100^{\circ} \mathrm{C}\right.$ for $5 \mathrm{~min}$ and then $24^{\circ} \mathrm{C}$ for a further 3 min). Subsequently, sections were treated with $3 \% \mathrm{H}_{2} \mathrm{O}_{2}$ for $10 \mathrm{~min}$ at room temperature to block endogenous peroxidase. The sections were washed with PBS three times for $10 \mathrm{~min}$ each and then blocked with $10 \%$ normal goat serum (Beijing Solarbio Science \& Technology Co., Ltd.) for $30 \mathrm{~min}$ at room temperature. The tissue sections were subsequently incubated with anti-CCR9 (1:200; cat. no. ab1662; Abcam), anti-vascular endothelial growth factor (VEGF; 1:200; cat. no. AF0312; Beyotime Institute of Biotechnology) anti-matrix metalloproteinase (MMP)-1 (1:500; cat. no. ab137332; Abcam) or anti-MMP-7 (1:500; cat. no. ab205525; Abcam) antibodies overnight at $4^{\circ} \mathrm{C}$. The slides were then incubated with horseradish peroxidase (HRP)-conjugated goat anti-mouse antibody (1:50; cat. no. A0216, Beyotime Institute of Biotechnology) or HRP-conjugated goat-anti-rabbit antibody (1:50; cat. no. A0208; Beyotime Institute of Biotechnology) at room temperature for $60 \mathrm{~min}$. Finally, the sections were washed with PBS, then stained with diaminobenzidine and hematoxylin (Beyotime Institute of Biotechnology) at room temperature for $5 \mathrm{~min}$. The sections were observed under a light microscope (magnification, x200; BX-43; Olympus Corporation). A semi-quantitative scoring system (15) was applied to assess the expression of CCR9, VEGF, MMP-1 and MMP-7 in the specimens based on the average intensity and density of positively stained cells by IHC. Staining intensity was graded as follows: i) 0 , no staining; ii) 1 , weak staining; iii) 2 , moderate staining; and iv) 3 , strong staining. The density of positively stained cells was scored according to the following criteria: i) $0, \leq 5 \%$; ii) $1,5-25 \%$; iii) 2 , $26-50 \%$; and iv) $3,>50 \%$. The total IHC score was calculated by multiplying the intensity score by the density score. A total IHC score $>0$ was defined as positive expression and a total IHC score $=0$ was defined as negative expression.

Cell culture. The NSCLC cell lines SK-MES-1 [squamous cell carcinoma (SCC)] and A549 [adenocarcinoma (AC)] were purchased from The Kunming Institute of Zoology, Chinese Academy of Sciences. Cells were cultured in RPMI-1640 medium (Gibco; Thermo Fisher Scientific, lnc.) containing 10\% FBS (Gemini Bio Products), $100 \mathrm{U} / \mathrm{ml}$ penicillin and $100 \mu \mathrm{g} / \mathrm{ml}$ streptomycin in $5 \% \mathrm{CO}_{2}$ at $37^{\circ} \mathrm{C}$. Before migration and invasion studies, lung cancer cells were cultured for $24 \mathrm{~h}$ in RPMI-1640 with $2 \%$ FBS.

Reverse transcription-quantitative PCR (RT-qPCR) primer design. The human mRNA sequences for VEGF-C, VEGF-D, MMP-1, MMP-7 and GAPDH were obtained from The National Institutes of Health National Center for Biotechnology Information (Genbank Database Accession nos. NM_005429.4, NM_004469.4, NM_001145938.2, NM_002423.5 and BC004109, respectively). These sequences were used to design primers for RT-qPCR analysis. The primer sequences were as follows: VEGF-C forward, 5'-AGCACG AGCTACCTCAGCAAGAC-3' and reverse, 5'-TTTAGACAT GCATCGGCAGGAA-3'; VEGF-D forward, 5'-AGCTGC CTGATGTCAACTGCTTA-3' and reverse, 5'-GTTCATTAC TGGAGCCCTGCAC-3'; MMP-1 forward 5'-AAGAATGAT GGGAGGCAAGT-3' and reverse, 5'-GGTTTCAGCATCTGG TTTCC-3'; MMP-7 forward 5'-GAGTGAGCTACAGTGGGA ACA-3' and reverse, 5'-CTATGACGCGGGAGTTTAACAT-3' and GAPDH forward, 5'-GACGGCAAGATGCACATCAC-3' and reverse, 5'-GAGATGTAGCACGGGATCATGG-3'.

RNA isolation and RT-qPCR analysis. Total RNA was isolated from lung cancer cells using the RNA Simple Total RNA kit (Tiangen Biotech Co., Ltd.). Cells were untreated, CCL25-treated or CCL25-treated and stained with anti-CCR9 antibody, cDNA was generated using the Quantscript RT kit (Tiangen Biotech Co., Ltd.) according to the manufacturer's protocols. Real-time RT-qPCR was performed using a 2X Taq PCR MasterMix (Tiangen Biotech Co., Ltd.) in a ABI7500 instrument (Applied Biosystems; Thermo Fischer Scientific, Inc.) using the following thermocycling conditions: Initial denaturation for $3 \mathrm{~min}$ at $94^{\circ} \mathrm{C}$, followed by 30 cycles of $94^{\circ} \mathrm{C}$ for $30 \mathrm{sec}$, $55^{\circ} \mathrm{C}$ for $30 \mathrm{sec}$ and $72^{\circ} \mathrm{C}$ for $1 \mathrm{~min}$, prior to final extension at $72^{\circ} \mathrm{C}$ for $5 \mathrm{~min}$. The expression of GAPDH was measured in each sample as an endogenous control. The ratio of 
mRNA expression was calculated for each group using the $2^{-\Delta \Delta \mathrm{Cq}}$ method (16).

Western blot analysis. The A549 and SK-MES-1 cell lines were treated with $100 \mathrm{ng} / \mathrm{ml} \mathrm{CCL25}$ (cat. no. 300-45; PeproTech, Inc.) or CCL25 (100 ng/ml) with $5 \mu \mathrm{g} / \mathrm{ml}$ anti-CCR9 antibody (cat. no. ab1662; Abcam) at $37^{\circ} \mathrm{C}$ and $5 \% \mathrm{CO}_{2}$ for $48 \mathrm{~h}$. Untreated cells were used as controls. Total protein from untreated and treated cells was washed with cold PBS and isolated in modified radio-immunoprecipitation assay buffer (50 mM Tris- $\mathrm{Cl}$ at $\mathrm{pH} 7.5 ; 150 \mathrm{mM} \mathrm{NaCl} ; 1 \% \mathrm{NP}-40 ; 1.0 \%$ sodium deoxycholate; $1 \%$ PMSF and $0.1 \%$ SDS) and quantified using the bicinchoninic acid method (Beyotime Institute of Biotechnology). Proteins were loaded (100 $\mu \mathrm{g}$ per lane) and resolved by SDS-PAGE on $10 \%$ gels using Tris-glycine-SDS buffer (25 mM Tris; $192 \mathrm{mM}$ glycine and 0.1\% SDS; pH 8.8). Proteins were transferred to polyvinylidene fluoride membranes (EMD Millipore) using a wet transfer apparatus (Bio-Rad Laboratories, Inc.). Membranes were incubated in blocking buffer (5\% non-fat milk dissolved in TBS $+0.1 \%$ Tween-20) for $1 \mathrm{~h}$ at room temperature. After blocking, membranes were incubated with anti-VEGF-C (1:500; cat. no. ab83905; Abcam), anti-VEGF-D (1:800; cat. no. ab155288; Abcam), anti-MMP-1 (1:1,000; cat. no. ab137332; Abcam), anti-MMP-7 (1:1,000; cat. no. ab205525; Abcam) and anti-GAPDH (1:2,000; cat. no. ab8245; Abcam) antibodies overnight at $4^{\circ} \mathrm{C}$. Membranes were then washed in wash buffer $(\mathrm{TBS}+0.1 \%$ Tween-20) three times for $10 \mathrm{~min}$ each time. After washing, membranes were incubated with the secondary antibodies for $2 \mathrm{~h}$ at $37^{\circ} \mathrm{C}$. The secondary antibodies were horseradish peroxidase-conjugated goat anti-rabbit antibody $(1: 8,000$; cat. no. ab205718; Abcam) or goat-anti-mouse antibody (1:8,000; cat. no. ab205719; Abcam). Following incubation, membranes were washed five times with washing buffer for $6 \mathrm{~min}$ each time. The immunoreactive bands were detected using the ECL Plus reagent (Beyotime Institute of Biotechnology). ImageJ software (version 1.5.2; https://imagej. nih.gov/ij) was used to quantify the data from three independent experiments. Bands from untreated cells were given a value of 1.0 and the protein levels of treated groups were normalized to this value.

Migration and invasion assays. Migration and invasion studies

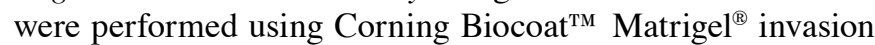
chambers with $8-\mu \mathrm{m}$ pore size (Corning, Inc.). Serum-free RPMI-1640 was added to the bottom and top chambers and allowed to hydrate the membrane for $2 \mathrm{~h}$ at $37^{\circ} \mathrm{C}$ with $5 \% \mathrm{CO}_{2}$. Subsequently, $1 \times 10^{4}$ cells were seeded to the top chamber and $100 \mathrm{ng} / \mathrm{ml}$ CCL25 (PeproTech, Inc.) was added into the bottom chamber as a chemo-attractant. To determine if the migration and invasion of lung cancer cells was mediated specifically by CCL25/CCR9 interaction, cells pre-incubated with $5.0 \mu \mathrm{g} / \mathrm{ml}$ anti-CCR9 antibody (Abcam) were added to the top chamber and allowed to migrate or invade in the presence of CCL25 at $37^{\circ} \mathrm{C}$ with $5 \% \mathrm{CO}_{2}$ overnight. After incubation, non-migrating cells in the upper surface of the membrane were removed. Cells at the bottom surface of the insert were fixed with $100 \%$ methanol for $10 \mathrm{~min}$ at room temperature, stained for 2 min with crystal violet (Beijing Solarbio Science $\&$ Technology Co., Ltd.) at room temperature and washed twice with deionized water. Migrated or invaded cells were counted using a light microscope (magnification, x200). All experiments were repeated three times for validation.

Bioinformatics analysis. The Kaplan-Meier Plotter online tool (http://kmplot.com/analysis/index. php?p=service\&cancer=lung) was used to investigate the association between CCR9 and CCL25 mRNA expression and clinical prognosis for patients with $\operatorname{NSCLC~}(n=1,926)(17)$. In this database (http://kmplot.com), multiple independent transcriptomic datasets, generated using caBIG (http://cabig. cancer.gov), GEO (http://www.ncbi.nlm.nih.gov/geo/) and TCGA (http://cancergenome.nih.gov), are merged and the gene expression data and survival information were analysed, where the hazard ratio (HR), 95\% confidence interval (CI) and log-rank P-value was determined and displayed. $\mathrm{P}<0.05$ was considered to indicate a statistically significant difference. The mutational level of CCR9 and CCL25 was determined using the cBioPortal for Cancer Genomics website (http://www. cbioportal.org/).

Statistical analysis. All values are presented as the mean \pm SD. Associations between CCR9 expression and clinicopathological parameters were assessed using the $\chi^{2}$ test as well as the Fisher's exact test. Statistically significant differences between groups were analyzed by one-way ANOVA with Tukey's post hoc test. Data were analyzed using SPSS 20.0 software (IBM Corp.) and GraphPad Prism 7 (GraphPad Software, Inc.). P $<0.05$ was used to indicate a statistically significant difference.

\section{Results}

CCR9, VEGF, MMP-1 and MMP-7 expression in NSCLC tissue. CCR9, VEGF, MMP-1 and MMP-7 were located on the cell membrane and/or the cytoplasm in the cancer tissues (Fig. 1). The positive rates of CCR9, VEGF, MMP-1 and MMP-7 were significantly higher in the lung cancer tissues compared with the adjacent normal tissues (Table I).

Association between CCR9 expression and clinical parameters in NSCLC. The CCR9 positive rate was higher in AC than SCC tissues. Additionally, CCR9 positivity was also significantly higher in the presence of lymph node metastasis. Moreover, CCR9 positive rates were significantly higher in VEGF, MMP-1 or MMP-7-positive tissue, compared with VEGF, MMP-1 or MMP-7-negative tissue, respectively. However, no significant difference in CCR9 expression was observed when patients were stratified by sex or age (Table II).

CCL25 induces the migration and invasion of NSCLC cells. A549 and SK-MES-1 cells lines demonstrated increased proliferative ability compared with the control $(\mathrm{P}<0.05)$ when treated with $100 \mathrm{ng} / \mathrm{ml}$ recombinant CCL25. Both A549 and SK-MES-1 cells were highly responsive in both migration and invasion assays (Fig. 2). Notably, the chemo-attractant effect of CCL25 was specific to CCR9 as demonstrated by the anti-CCR9 antibody blocking the chemo-attracttant effect (Fig. 2; $\mathrm{P}<0.05$ ).

CCL25 modulates VEGF-C, VEGF-D, MMP-1 and MMP-7 expression in NSCLC cells. At the transcriptional and protein 

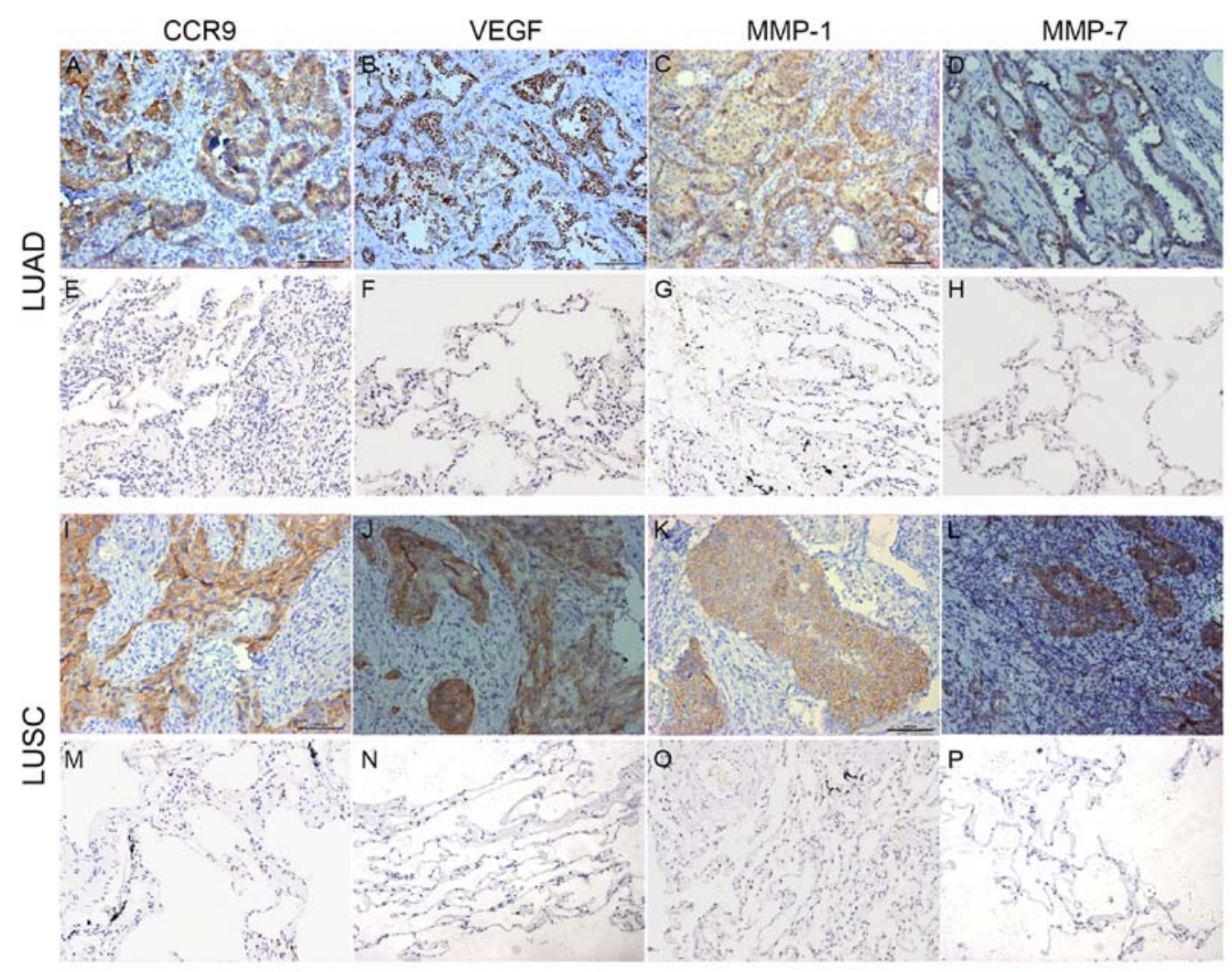

Figure 1. CCR9, VEGF, MMP-1 and MMP-7 expression in the non-small cell lung cancer and adjacent normal tissues. Positive (A) CCR9, (B) VEGF, (C) MMP-1 and (D) MMP-7 expression in LUAD tissue. Negative (E) CCR9, (F) VEGF, (G) MMP-1 and (H) MMP-7 expression in lung adjacent normal tissue. Positive (I) CCR9, (J) VEGF, (K) MMP-1 and (L) MMP-7 expression in LUSC tissue. Negative (M) CCR9, (N) VEGF, (O) MMP-1 and (P) MMP-7 expression in lung adjacent normal tissue. Magnification x200. CCR9, CC chemokine receptor 9; VEGF, vascular endothelial growth factor; MMP-1, matrix metalloproteinase-1; MMP-7, matrix metalloproteinase-7; LUAD, lung adenocarcinoma; LUSC, lung squamous cell carcinoma.

level, the expressions of VEGF-C, VEGF-D, MMP-1 and MMP-7 were upregulated in the A549 and SK-MES-1 cell lines after CCL25 stimulation (Figs. 3 and 4; $\mathrm{P}<0.05$ in all cases). Furthermore, CCL25-induced upregulation was reversed by anti-CCR9 antibody treatment (Figs. 3 and 4; $\mathrm{P}<0.05$ in all cases). However, MMP-7 protein expression was not modified by CCL25 or anti-CCR9 antibody in the SK-MES-1 cells $(\mathrm{P}>0.05)$.

Survival analysis of CCR 9 and CCL25 in patients with NSCLC. The association between CCR9 and CCL25 positive rates, and clinical prognosis in patients with NSCLC $(n=1,926)$ was examined using a Kaplan-Meier analysis. Patients with lower CCR9 expression demonstrated better overall survival (OS), compared with those with higher CCR9 expression (HR=1.14; $\mathrm{P}=0.042$; Fig. 5A). In addition, patients with lower CCL25 expression had better OS, compared with those with higher CCL25 expression (HR=1.13; $\mathrm{P}=0.05$; Fig. 5B). Although the results presented in Fig. 5A are only marginally statistically significant, the actual OS benefits of low CCR9 and CCL25 expression in months are substantial. Indeed, in the case of CCR9, the OS benefit for high expression, compared with low expression, was as follows: i) 50 months, 357 compared with 471; ii) 100 months, 72 compared with 131; iii) 150 months, 11 compared with 46; and iv) 200 months, 1 compared with 6. In the case of CCL25, the OS benefit for high expression, compared with low expression, was as follows: i) 50 months, 354 compared with 474 ; ii) 100 months, 80 compared with
123; iii) 150 months, 14 compared with 43; and iv) 200 months, 1 compared with 6 . Moreover, in a sub-group analysis, low levels of CCR9 mRNA were significantly associated with an improved OS for patients who never smoked (Fig. 5C; $\mathrm{HR}=2.22 ; \mathrm{P}<0.01)$. However, there was no similar association between the expression of CCL25 mRNA and OS among patients who never smoked (Fig. 5D; HR=1.49; $\mathrm{P}=0.16$ ). Furthermore, low levels of both CCR9 and CCL25 mRNA were indicative of a favorable OS outcome among patients who smoked (Fig. 5E and F).

Mutation analysis. The mutation status of CCR9 and CCL25 in lung tumor tissues was examined using the public database of cBioPortal for Cancer Genomics (Fig. 6). As presented in Fig. 6B, CCR9 had a somatic mutation rate of $0.26 \%$ in NSCLC $(3 / 1,144)$ in a TCGA 2016 study $(18), 0.55 \%$ in lung AC (1/183) in the Imielinski et al (19) study. For lung SCC, the CCR9 mutational rates were found to be $0.62(3 / 487)$, $0.56(1 / 178)$ and $0.2 \%(1 / 511)$ in TCGA PanCan (20), TCGA pub (21) and TCGA studies (http://gdac.broadinstitute. org/runs/stddata_2016_01_28/data/LUSC/20160128/gdac.broad institute.org_LUSC.Mutation_Packager_Calls.Level_3.20160 12800.0.0.tar.gz), respectively.

CCL25 had a somatic mutation rate of $0.17 \%(2 / 1,144)$ in NSCLC data from TCGA 2016 study and $0.53 \%(3 / 566)$ in lung AC data from TCGA PanCan study (Fig. 6C). Altogether, these results suggested that mutation of CCR9 and CCL25 is 
Table I. Association between CCR9 and clinicopathological characteristics.

\begin{tabular}{|c|c|c|c|c|}
\hline Clinicopathological characteristic & Number of patients & CCR9 postivity $[\mathrm{N},(\%)]$ & $\chi^{2}$ & P-value \\
\hline \multicolumn{5}{|l|}{ Sex } \\
\hline Male & 40 & $25(62.50)$ & \multirow[t]{2}{*}{0.330} & \multirow[t]{2}{*}{0.566} \\
\hline Female & 20 & $14(70.00)$ & & \\
\hline \multicolumn{5}{|l|}{ Age (years) } \\
\hline$\leq 60$ & 37 & $22(59.46)$ & \multirow[t]{2}{*}{1.302} & \multirow[t]{2}{*}{0.254} \\
\hline$>60$ & 23 & $17(73.91)$ & & \\
\hline \multicolumn{5}{|l|}{ Histology } \\
\hline $\mathrm{SCC}$ & 25 & $12(48.00)$ & \multirow[t]{2}{*}{5.444} & \multirow[t]{2}{*}{0.020} \\
\hline $\mathrm{AC}$ & 35 & $27(77.14)$ & & \\
\hline \multicolumn{5}{|l|}{ Lymph node metastasis } \\
\hline No & 15 & $4(26.67)$ & \multirow[t]{2}{*}{12.918} & \multirow[t]{2}{*}{$<0.001$} \\
\hline Yes & 45 & $35(77.78)$ & & \\
\hline \multicolumn{5}{|l|}{ VEGF } \\
\hline Positive & 31 & $25(80.65)$ & \multirow[t]{2}{*}{6.901} & \multirow[t]{2}{*}{0.008} \\
\hline Negative & 29 & $14(48.28)$ & & \\
\hline \multicolumn{5}{|l|}{ MMP-1 } \\
\hline Positive & 36 & $27(75.00)$ & \multirow[t]{2}{*}{3.956} & \multirow[t]{2}{*}{0.047} \\
\hline Negative & 24 & $12(50.00)$ & & \\
\hline \multicolumn{5}{|l|}{ MMP-7 } \\
\hline Positive & 33 & $29(87.88)$ & \multirow[t]{2}{*}{16.873} & \multirow[t]{2}{*}{$<0.001$} \\
\hline Negative & 27 & $10(37.04)$ & & \\
\hline
\end{tabular}

SCC, squamous cell carcinoma; AC, adenocarcinoma; CCR9, CC chemokine receptor 9; VEGF, vascular endothelial growth factor; MMP, matrix metalloproteinase.

Table II. Expression of CCR9, VEGF, MMP-1 and MMP-7 in tumor tissues and adjacent normal tissues in patients with non-small cell lung cancer.

\begin{tabular}{|c|c|c|c|c|}
\hline Parameter & Tumour tissue, n (\%) & Adjacent normal tissue, n (\%) & $\chi^{2}$ & P-value \\
\hline CCR9 & $39(65.00)$ & $15(25.00)$ & 19.394 & $<0.0001$ \\
\hline VEGF & $31(51.67)$ & $10(16.67)$ & 16.338 & $<0.0001$ \\
\hline MMP-1 & $36(60.00)$ & $11(18.33)$ & 21.860 & $<0.0001$ \\
\hline MMP-7 & $33(55.00)$ & $6(10.00)$ & 27.693 & $<0.0001$ \\
\hline
\end{tabular}

CCR9, CC chemokine receptor 9; VEGF, vascular endothelial growth factor; MMP, matrix metalloproteinase.

a rare occurrence in patients with NSCLC. This is in stark contract with epidermal growth factor receptor (EGFR), which has a mutational rate of $\sim 10 \%$ in Caucasian patients with NSCLC and $\leq 50 \%$ of Asian patients with NSCLC (22-24), or ALK receptor tyrosine kinase (ALK) with a 3-5\% rate in patients with NSCLC (25).

\section{Discussion}

In the present study, the CCL25/CCR9 signaling axis was demonstrated to regulate the expression of VEGF-C, VEGF-D, MMP-1 and MMP-7, and may promote the invasion and migration of the lung cancer cells. Survival analysis demonstrated that patients with lower expression of CCL25 or CCR9 in their tumors displayed better prognosis.

Chemokines are known mediators of leukocyte trafficking and host defense (26), Indeed, previous studies have determined that the involvement of chemokine receptors is of importance in patient prognosis (27), apoptosis (28) and metastatic (29) signaling machinery in various cancer types. Among all the chemokine receptors, studies on the role of CXCR4 have been more extensive. These previous studies suggested that CXCR4 $(8,12,30-32)$ was highly expressed in NSCLC, and functional blockade of this interaction could inhibit metastasis to the bone marrow, lymph nodes or pleural space. These findings highlight the effect of 


\section{A}
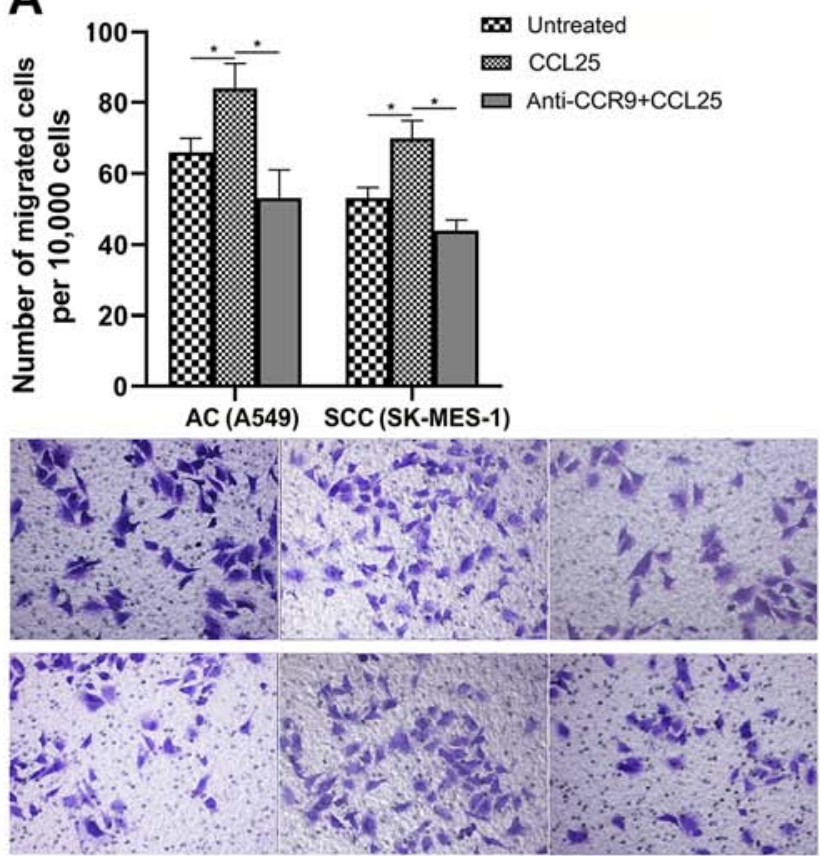
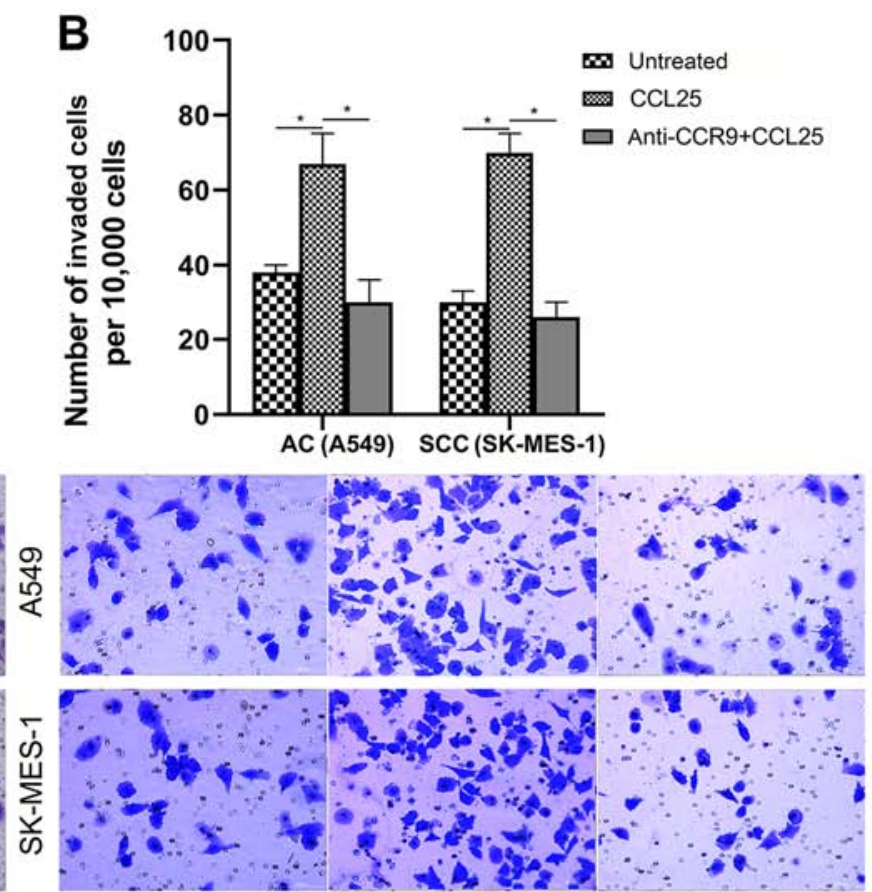

Figure 2. Migration and invasion of A549 and SK-MES-1 cell lines (). (A) Number of migrating cells was significantly increased after CCL25 treatment and the addition of an anti-CCR9 antibody could significantly abrogate this increase. (B) Number of invading cells was significantly increased after CCL25 treatment and the addition of an anti-CCR9 antibody significantly inhibited this increase. Magnification, x 200. ${ }^{*} \mathrm{P}<0.05$. CCR9, CC chemokine receptor 9; CCL25, CC motif chemokine ligand 25; AC, adenocarcinoma; SCC, squamous cell carcinoma.
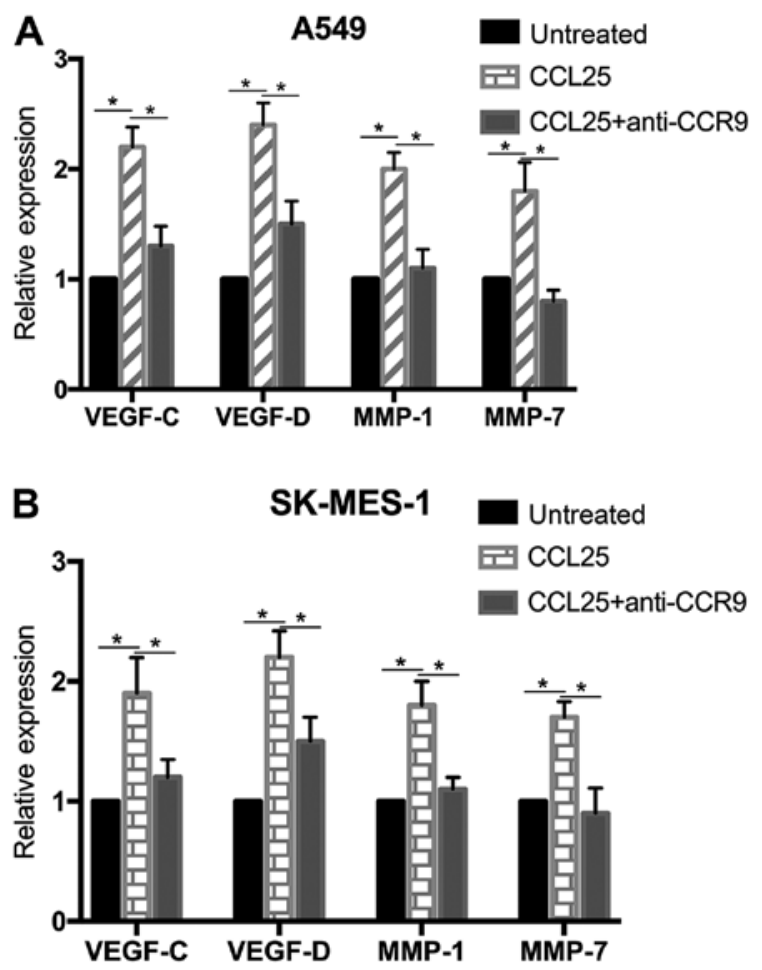

Figure 3. mRNA expression of VEGF-C, VEGF-D, MMP-1 and MMP-7 in non-small cell lung cancer cell lines. (A) Expression of VEGF-C, VEGF-D, MMP-1 and MMP-7 in A549 cells significantly increased after CCL25 treatment, and the addition of an anti-CCR9 antibody inhibited CCL25-mediated upregulation. (B) Expression of VEGF-C, VEGF-D, MMP-1 and MMP-7 in SK-MES-1 cells increased after CCL25 treatment, and the anti-CCR9 antibody abrogated this CCL25-mediated increase. ${ }^{*} \mathrm{P}<0.05$. CCR9, CC chemokine receptor 9; CCL25, CC motif chemokine ligand 25; VEGF, vascular endothelial growth factor; MMP-1, matrix metalloproteinase-1; MMP-7, matrix metalloproteinase-7. chemokine/chemokine receptor signaling on the metastatic potential of NSCLC.

Several steps are required to achieve metastasis, including active migration, extracellular matrix degradation and adhesion to vascular endothelial cells. Migration and invasion associated with metastatic potential can be triggered by chemokine binding to chemokine receptor on the cell surface $(33,34)$. Tumor lymphangiogenesis was previously found to be associated with the VEGF-C/VEGF-D/VEGF receptor-3 (VEGFR-3) signaling axis $(35,36)$. Previous studies have determined VEGF-C activates VEGFR-3, which in turn promotes proliferation (37), migration (38) and apoptosis protection (39). In various cancer types, the tumor cells produce VEGF-C and recruit monocytes or macrophages into tumor tissue (40). These monocytes and macrophages differentiate to M2-polarized tumor-associated macrophages, which also produce VEGF-C, and further increase lymphatic vessel development (41). Moreover, lymph-angiogenic factors derived from normal lymphatic cells can reprogram the gene expression profile of these cells and convert them to tumor-derived lymphatic cells during tumor development and progression (42). Tumor-derived lymphatic cells express specific lymphatic markers, such as VEGFR-3 and lymphatic vessel endothelial receptor 1 , and form a lymphatic system in vivo (43).

VEGF-C and VEGF-D, members of the VEGF family, have been demonstrated to stimulate the proliferation of lymphatic endothelial cells, and to promote lymphatic invasion and lymph node metastasis through VEGFR-3 signaling, which is critical for the growth of lymphatic vessels $(44,45)$. These findings provided theoretical evidence for the role of VEGF-C and VEGF-D in promoting cancer metastasis (46). 
A

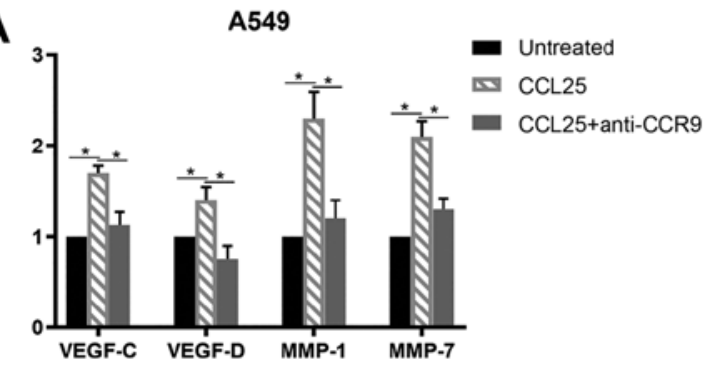

B

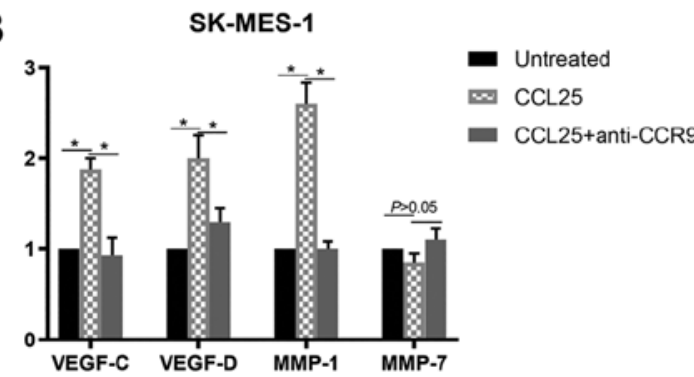

C
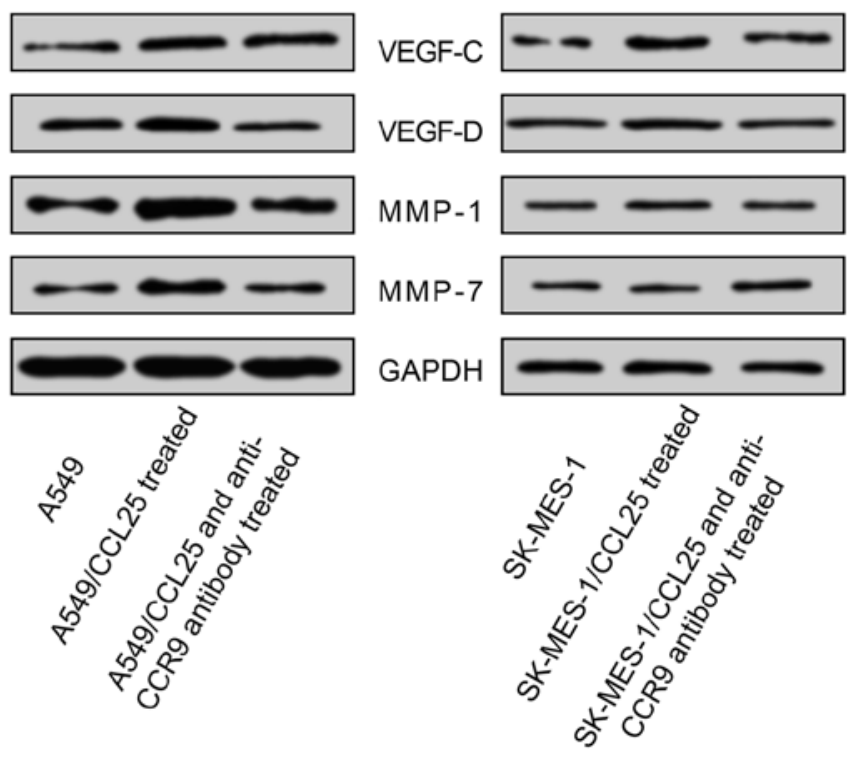

Figure 4. Protein expression of VEGF-C, VEGF-D, MMP-1 and MMP-7 in non-small cell lung cancer cell lines. (A) Expression of VEGF-C, VEGF-D, MMP-1 and MMP-7 in A549 cells increased following CCL25 treatment and the addition of an anti-CCR9 antibody abrogated this upregulation. (B) Expression of VEGF-C, VEGF-D and MMP-1 in SK-MES-1 cells increased following CCL25 treatment. Addition of anti-CCR9 could abrogate this increase. (C) Protein expression of VEGF-C, VEGF-D, MMP-1 and MMP-7 was examined in A549 and SK-MES-1 cells. "P<0.05. CCR9, CC chemokine receptor 9; CCL25, CC motif chemokine ligand 25; VEGF, vascular endothelial growth factor; MMP-1, matrix metalloproteinase-1; MMP-7, matrix metalloproteinase-7.

A

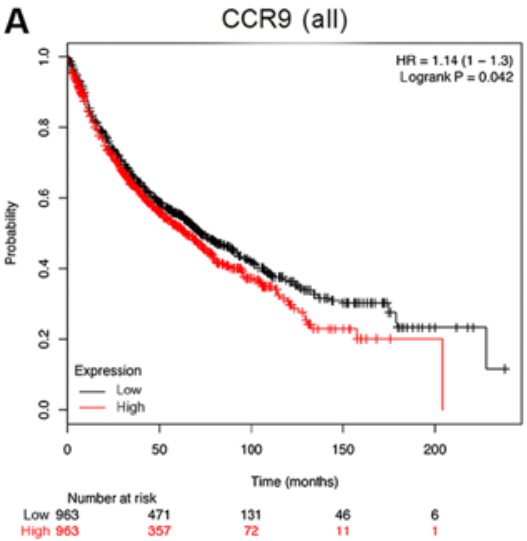

D

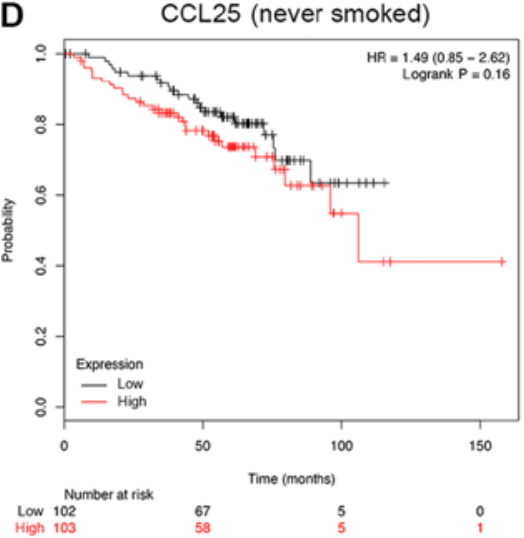

B

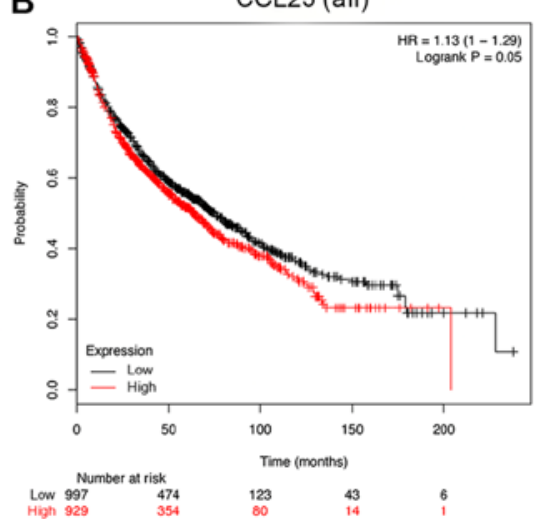

$\mathrm{E}$

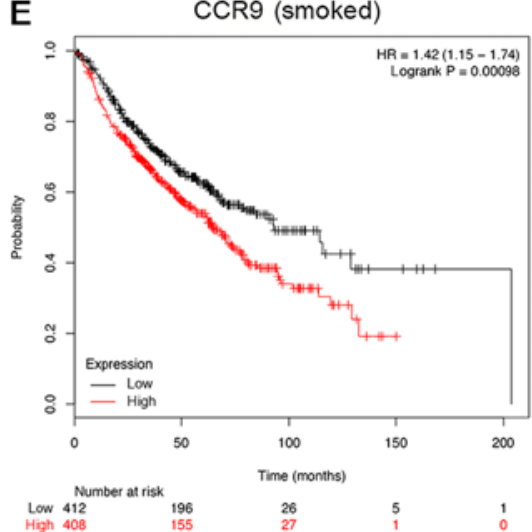

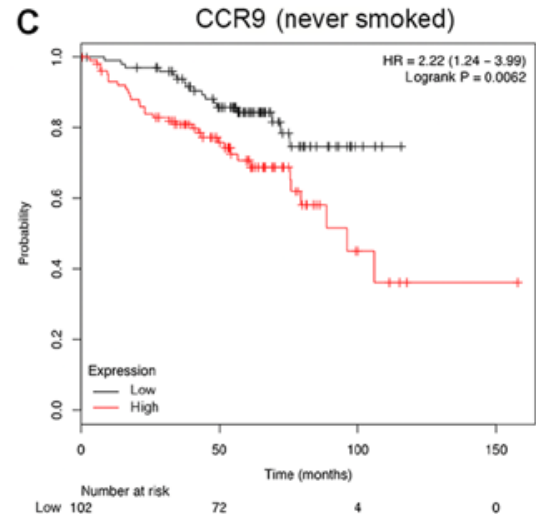

$\mathbf{F}$

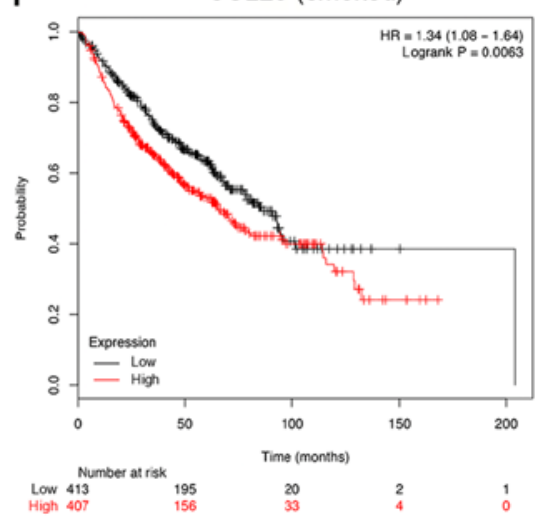

Figure 5. Survival analysis of patients with NSCLC according to CCL25 and CCR9 expression. Kaplan-Meier survival plots showing that high expression of CCR9 or CCL25 is associated with poor survival in patients with NSCLC. (A) Association between CCR9 mRNA expression and OS. (B) Association between CCL25 mRNA expression and OS. (C) Association between CCR9 mRNA expression and OS among patients who never smoked. (D) Association between CCL25 mRNA expression and OS among patients who never smoked. (E) Association between CCR9 mRNA expression and OS among patients who smoked. (F) Association between CCL25 mRNA expression and OS among patients who smoked. NSCLC, non-small cell lung cancer; CCR9, CC chemokine receptor 9; CCL25, CC motif chemokine ligand 25; OS, overall survival. 


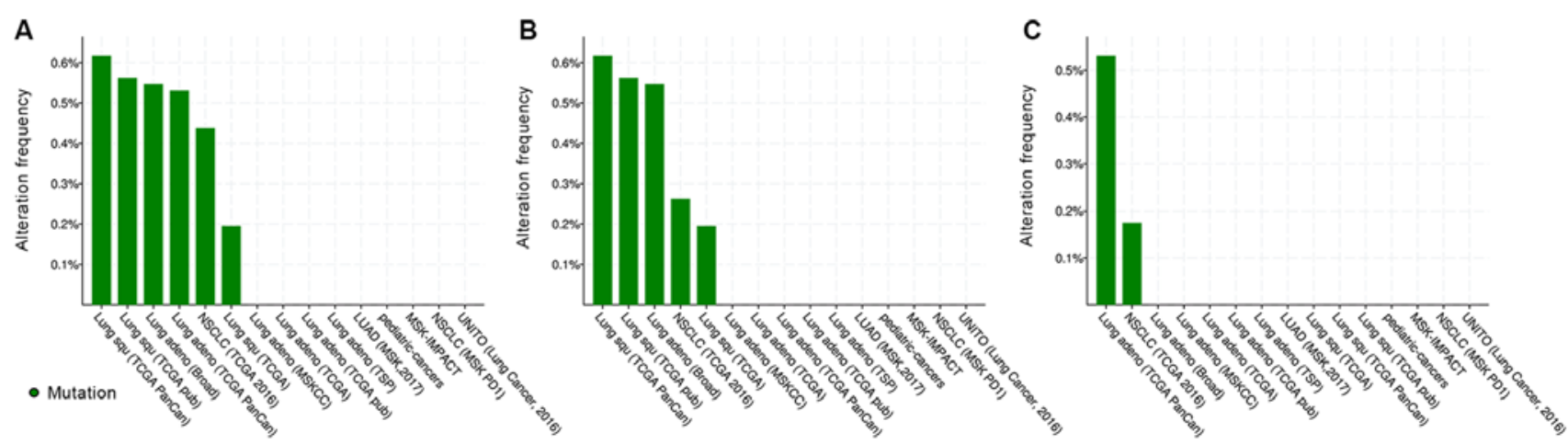

Figure 6. Mutation analysis of CCR9 and CCL25 through the public database of the cBioPortal for Cancer Genomics. The data are from studies compiled from the cBioPortal for Cancer Genomics public database. Title of the study is presented on the $\mathrm{x}$-axis and the alteration frequency (mutation frequency) on the y-axis. (A) Combined mutational frequency of CCR9 and CCL25. (B) Mutation frequency of CCR9. (C) Mutation frequency of CCL25. CCR9, CC chemokine receptor 9; CCL25, CC motif chemokine ligand 25.

In the present study, VEGF expression was higher in NSCLC tumors compared with the tumor-adjacent normal tissues, and CCL25 treatment upregulated the expression of VEGF-C and -D in lung cancer cell lines, which could be blocked by the anti-CCR9 antibody. The aforementioned results were consistent with previous cancer-related studies on $\operatorname{VEGF}(47,48)$.

In addition to VEGF-C and -D, the metalloproteinases MMP-1 and MMP-7, which serve as predominant components in the degradation of collagenous extracellular matrix, play a critical role in cancer biology, including cell migration, invasion and angiogenesis $(49,50)$. Previous studies (51-53) have demonstrated an important role for MMP-1 and MMP-7 in the development of various cancer types; however, the effect of MMP-1 and MMP-7 have not been well characterized in NSCLC. In the present study, it was observed that the migration and invasion of lung cancer cells was enhanced by CCL 25 treatment. This provides insight on a potential association between the CCL25/CCR9 interaction, and the migration and invasion of lung cancer cells. CCL25 was identified to influence the secretion of VEGF-C, VEGF-D, MMP-1 and MMP-7 in NSCLC cells, which influenced migratory and invasive potential in vitro. In addition, low expression of CCR9 and CCL25 is associated with good OS in NSCLC. In consideration of our previous study (14) and the present study, the CCL25/CCR9 axis may promote the invasion and migration of cancer cells through modulation of VEGF-C, VEGF-D, MMP-1 and MMP-7.

In the present study, another notable result was the low mutation of CCR9 and CCL25, compared with EGFR. For clinical therapy, the effectiveness of either ALK or EGFR inhibitors is restricted by the eventual occurrence of drug resistance despite the initial promising responses. Considering the low mutation rates of CCL25 and CCR9 in NSCLC, future research should focus on targeting the CCL25/CCR9 axis.

There are several limitations in the present study. Firstly, only a blocking antibody was used to validate the expression of CCL25 and CCR9 on lung cancer cell lines. Additional methods of altering the signaling pathway, such as with gene expression manipulation with lentivirus, for example, would additionally be informative. In vivo animal experiments would also be valuable in helping to understand this signaling pathway in lung cancer cells. Investigating the effect of CCL25/CCR9 signaling on transcription factor interactions using chromatin immunoprecipitation analysis could also improve the understanding of the pathway in lung cancer cells. Finally, the OS benefit of CCL25 may be re-evaluated by expanding the number of samples, and to examine the potential reasons for the different OS benefits observed between CCR9 and CCL25 in the future.

\section{Acknowledgements}

Not applicable.

\section{Funding}

The present study was supported by The Research Projects of Shanghai Health and Planning Commission (grant no. 201740015); Shanghai Municipal Natural Science Foundation (grant no. 18ZR1413000); and Natural Science Foundation of China (grant no. 81560383).

\section{Availability of data and materials}

The datasets used and/or analyzed during the present study are available from the corresponding author on reasonable request.

\section{Authors' contributions}

YN and DT wrote the manuscript and analyzed the data. YN and LF performed the experiments. YN contributed to the conceptual idea for the manuscript. WG and HL contributed to study design and manuscript editing. All authors read and approved the final manuscript.

\section{Ethics approval and consent to participate}

The present study protocol was approved by The Ethics Committee Review Board of The Huadong Hospital Affiliated with Fudan University (approval no. 20180046), and written informed consent was obtained from every participant. 


\section{Patient consent for publication}

Not applicable.

\section{Competing interests}

The authors declare that they have no competing interests.

\section{References}

1. Siegel RL, Miller KD and Jemal A: Cancer statistics, 2019. CA Cancer J Clin 69: 7-34, 2019.

2. Fidler IJ: Critical factors in the biology of human cancer metastasis: Twenty-eighth GHA Clowes memorial award lecture. Cancer Res 50: 6130-6138, 1990.

3. Fidler IJ: Critical determinants of cancer metastasis: Rationale for therapy. Cancer Chemother Pharmacol 43 (43 Suppl): S3-S10, 1999.

4. Strieter RM: Chemokines: Not just leukocyte chemoattractants in the promotion of cancer. Nat Immunol 2: 285-286, 2001.

5. Stein JV and Nombela-Arrieta C: Chemokine control of lymphocyte trafficking: A general overview. Immunology 116: 1-12, 2005.

6. Olson TS and Ley K: Chemokines and chemokine receptors in leukocyte trafficking. Am J Physiol Regulatory Integrative Comparative Physiol 283: R7-R28, 2002.

7. Saintigny P, Massarelli E, Lin S, Ahn YH, Chen Y, Goswami S and Zhang L: CXCR2 expression in tumor cells is a poor prognostic factor and promotes invasion and metastasis in lung adenocarcinoma. Cancer Res 73: 571-582, 2013.

8. Su L, Zhang J, Xu H, Wang Y, Chu Y, Liu R and Xiong S: Differential expression of CXCR4 is associated with the metastatic potential of human non-small cell lung cancer cells. Clin Cancer Res 11: 8273-8280, 2005.

9. Hald SM, Kiselev Y, Al-Saad S, Richardsen E, Johannessen C, Eilertsen M and Bremnes RM: Prognostic impact of CXCL16 and CXCR6 in non-small cell lung cancer: Combined high CXCL16 expression in tumor stroma and cancer cells yields improved survival. BMC Cancer 15: 441, 2015.

10. Mukaida N, Sasaki SI and Baba T: Chemokines in cancer development and progression and their potential as targeting molecules for cancer treatment. Mediators Inflamm 2014: 170381, 2014.

11. Zhu YM, Webster SJ,FlowerD and Woll PJ: Interleukin-8/CXCL8 is a growth factor for human lung cancer cells. Br J Cancer 91: 1970,2004

12. Phillips RJ, Burdick MD, Lutz M, Belperio JA, Keane MP and Strieter RM: The stromal derived factor-1/CXCL12-CXC chemokine receptor 4 biological axis in non-small cell lung cancer metastases. Am J Respir Crit Care Med 167: 1676-1686, 2003.

13. Gupta P, Sharma PK, Mir H, Singh R, Singh N, Kloecker GH and Singh S: CCR9/CCL25 expression in non-small cell lung cancer correlates with aggressive disease and mediates key steps of metastasis. Oncotarget 5: 10170, 2014.

14. Li B, Wang Z, Zhong Y, Lan J, Li X and Lin H: CCR9-CCL25 interaction suppresses apoptosis of lung cancer cells by activating the PI3K/Akt pathway. Med Oncol 32: 66, 2015.

15. Zhong Y, Jiang L, Lin H, Li B, Lan J, Liang S, Shen B, Lei Z and Zheng W: Expression of $\mathrm{CC}$ chemokine receptor 9 predicts poor prognosis in patients with lung adenocarcinoma. Diagn Pathol 10: 101,2015.

16. Livak KJ and Schmittgen TD: Analysis of relative gene expression data using real-time quantitative PCR and the 2(-Delta Delta C(T)) method. Methods 25: 402-408, 2001.

17. Győrffy B, Surowiak P, Budczies J and Lánczky A: Online survival analysis software to assess the prognostic value of biomarkers using transcriptomic data in non-small-cell lung cancer. PLoS One 8: e82241, 2013.

18. Campbell JD, Alexandrov A, Kim J, Wala J, Berger AH, Pedamallu CS and Imielinski M: Distinct patterns of somatic genome alterations in lung adenocarcinomas and squamous cell carcinomas. Nat Genet 48: 607, 2016.

19. Imielinski M, Berger AH, Hammerman PS, Hernandez B, Pugh TJ, Hodis E and Sougnez C: Mapping the hallmarks of lung adenocarcinoma with massively parallel sequencing. Cell 150: 1107-1120,2012.

20. Ellrott K, Bailey MH, Saksena G, Covington KR, Kandoth C, Stewart C and Sofia HJ: Scalable open science approach for mutation calling of tumor exomes using multiple genomic pipelines. Cell Systems 6: 271-281, 2018.
21. Cancer Genome Atlas Research Network: Comprehensive genomic characterization of squamous cell lung cancers. Nature 489: 519, 2012.

22. Skov BG, Høgdall E, Clementsen P, Krasnik M, Larsen KR, Sørensen JB and Mellemgaard A: The prevalence of EGFR mutations in non-small cell lung cancer in an unselected Caucasian population. APMIS 123: 108-115, 2015.

23. Shi Y, Li J, Zhang S, Wang M, Yang S, Li N and Zheng M Molecular epidemiology of EGFR mutations in Asian patients with advanced non-small-cell lung cancer of adenocarcinoma histology-Mainland China subset analysis of the PIONEER study. PLoS One 10: e0143515, 2015.

24. Hirsch FR and Bunn PA Jr: EGFR testing in lung cancer is ready for prime time. Lancet Oncol 5: 432-433, 2009.

25. Haratake N, Seto T, Takamori S, Toyozawa R, Nosaki K, Miura N, Ohba T, Toyokawa G, Taguchi K, Yamaguchi M, et al: Short progression-free survival of ALK inhibitors sensitive to secondary mutations in ALK-positive NSCLC patients. Thorac Cancer 10: 1779-1787, 2019.

26. Zlotnik A and Yoshie O: Chemokines: A new classification system and their role in immunity. Immunity 12: 121-127, 2000.

27. Nishikawa G, Kawada K, Nakagawa J, Toda K, Ogawa R, Inamoto $\mathrm{S}$ and Sakai Y: Bone marrow-derived mesenchymal stem cells promote colorectal cancer progression via CCR5. Cell Death Dis 10: 264, 2019.

28. Zhu F, Liu Y, Liu L, Zhou Y, Zhou P, Yan Q and Ding S: CKLF1 enhances Inflammation-mediated carcinogenesis and prevents Doxorubicin-induced apoptosis via IL-6/STAT3 signaling in HCC. Clin Cancer Res 25: 4141-4154, 2019

29. Steele CW, Karim SA, Leach JD, Bailey P, Upstill-Goddard R, Rishi L and Eberlein C: CXCR2 inhibition profoundly suppresses metastases and augments immunotherapy in pancreatic ductal adenocarcinoma. Cancer Cell 29: 832-845, 2016.

30. OonakaharaKI,MatsuyamaW,HigashimotoI,KawabataM,ArimuraK and Osame M: Stromal-derived factor-1alpha/CXCL12-CXCR 4 axis is involved in the dissemination of NSCLC cells into pleural space. Am J Respir Cell Mol Biol 30: 671-677, 2004.

31. Yusen W, Xia W, Shengjun Y, Shaohui Z and Hongzhen Z: The expression and significance of tumor associated macrophages and CXCR4 in non-small cell lung cancer. J BUON 23: 398-402, 2018.

32. Choi YH, Burdick MD, Strieter BA, Mehrad B and Strieter RM: CXCR4, but not CXCR7, discriminates metastatic behavior in non-small cell lung cancer cells. Mol Cancer Res 12: 38-47, 2014.

33. Siegel G, Malmsten M and Klüssendorf D: Tumor cell locomotion and metastatic spread. Microsc Res Tech 43: 276-282, 1998.

34. Navolotski A, Rumjnzev A, Lü H, Proft D, Bartholmes P and Zänker KS: Migration and gap junctional intercellular communication determine the metastatic phenotype of human tumor cell lines. Cancer Lett 118: 181-187, 1997.

35. Achen MG and Stacker SA: Molecular control of lymphatic metastasis. Ann N Y Acad Sci 1131: 225-234, 2008.

36. Yonemura Y, Endo Y, Tabata K, Kawamura T, Yun HY, Bandou E, Sasaki T and Miura M: Role of VEGF-C and VEGF-D in lymphangiogenesis in gastric cancer. Int J Clin Oncol 10: 318-327, 2005

37. Marchiò S, Primo L, Pagano M, Palestro G, Albini A, Veikkola T, Cascone I, Alitalo K and Bussolino F: Vascular endothelial growth factor-C stimulates the migration and proliferation of Kaposi's sarcoma cells. J Biol Chem 274: 27617-27622, 1999.

38. Timoshenko AV, Rastogi S and Lala PK: Migration-promoting role of VEGF-C and VEGF-C binding receptors in human breast cancer cells. Br J Cancer 97: 1090-1098, 2007.

39. Zhao L, Zhu Z, Yao C, Huang Y, Zhi E, Chen H, Tian R, Li P, Yuan Q, Xue Y, et al: VEGFC/VEGFR3 signaling regulates mouse Spermatogonial cell proliferation via the activation of AKT/MAPK and cyclin D1 pathway and mediates the apoptosis by affecting caspase 3/9 and Bcl-2. Cell Cycle 17: 225-239, 2018.

40. Schmid MC and Varner JA: Myeloid cell trafficking and tumor angiogenesis. Cancer Lett 250: 1-8, 2007.

41. Mantovani A, Sozzani S, Locati M, Allavena P and Sica A: Macrophage polarization: Tumor-associated macrophages as a paradigm for polarized M2 mononuclear phagocytes. Trends Immunol 23: 549-555, 2002.

42. Movahedi K, Laoui D, Gysemans C, Baeten M, Stangé G, Van den Bossche, Mack M, Pipeleers D, In't Veld P, De Baetselier P, Van Ginderachter JA: Different tumor microenvironments contain functionally distinct subsets of macrophages derived from Ly6C(high) monocytes. Cancer Res 70: 5728-5739, 2010. 
43. Volk-Draper LD, Hall KL, Wilber AC and Ran S: Lymphatic endothelial progenitors originate from plastic myeloid cells activated by toll-like receptor-4. PLoS One 12: e0179257, 2017.

44. Alitalo A and Detmar M: Interaction of tumor cells and lymphatic vessels in cancer progression. Oncogene 31: 4499, 2012

45. Achen MG and Stacker SA: Vascular endothelial growth factor-D: Signaling mechanisms, biology, and clinical relevance. Growth Factors 30: 283-296, 2012.

46. Stacker SA, Achen MG, Jussila L, Baldwin ME and Alitalo K Metastasis: Lymphangiogenesis and cancer metastasis. Nature Rev Cancer 2: 573, 2002.

47. Chen Y, Liu Y, Wang Y, Li W, Wang X, Liu X, Chen Y, Ouyang C and Wang J: Quantification of STAT3 and VEGF expression for molecular diagnosis of lymph node metastasis in breast cancer. Medicine (Baltimore) 96: e8488, 2017.

48. Guo J, Lou W, Ji Y and Zhang S: Effect of CCR7, CXCR4 and VEGF-C on the lymph node metastasis of human pancreatic ductal adenocarcinoma. Oncol Lett 5: 1572-1578, 2013.

49. Sánchez-Lorencio MI, Saenz L, Ramirez P, Villalba-López F, de la Orden V, Mediero-Valeros B, Revilla Nuin B, Gonzalez MR, Cascales-Campos PA, Ferreras-Martínez D, et al: Matrix metalloproteinase 1 as a novel biomarker for monitoring hepatocellular carcinoma in liver transplant patients. Transplant Proc 50: 623-627, 2018
50. Gao Y, Nan X, Shi X, Mu X, Liu B, Zhu H, Yao B, Liu X, Yang T, Hu Y and Liu S: SREBP1 promotes the invasion of colorectal cancer accompanied upregulation of MMP7 expression and NF- $\kappa$ B pathway activation. BMC Cancer 19: 685, 2019.

51. Yokoi A, Yoshioka Y, Yamamoto Y, Ishikawa M, Ikeda SI, Kato T, Kiyono T, Takeshita F, Kajiyama H, Kikkawa F and Ochiya T: Malignant extracellular vesicles carrying MMP1 mRNA facilitate peritoneal dissemination in ovarian cancer. Nat Commun 8: 14470, 2017.

52. Sizemore ST, Sizemore GM, Booth CN, Thompson CL, Silverman P, Bebek G, Abdul-Karim FW, Avril S and Keri RA: Hypomethylation of the MMP7 promoter and increased expression of MMP7 distinguishes the basal-like breast cancer subtype from other triple-negative tumors. Breast Cancer Res Treat 146: 25-40, 2014.

53. Yuan S, Lin LS, Gan RH, Huang L, Wu XT, Zhao Y, Su BH, Zheng D and Lu YG: Elevated matrix metalloproteinase 7 expression promotes the proliferation, motility and metastasis of tongue squamous cell carcinoma. BMC Cancer 20: 33, 2020.

This work is licensed under a Creative Commons Attribution-NonCommercial-NoDerivatives 4.0 International (CC BY-NC-ND 4.0) License. 